\title{
Simple normal crossing varieties with prescribed dual complex
}

\author{
János Kollár
}

\begin{abstract}
We prove that every simplicial complex is the dual complex of some simple normal crossing divisor in a smooth variety. As an application, we simplify and extend earlier results on the existence of singularities with given dual complex.
\end{abstract}

\section{Introduction}

Let $W$ be a reducible variety with simple normal crossing singularities only. The combinatorial structure of $W$ is described by the dual complex $\mathcal{D}(W)$ whose vertices correspond to the irreducible components $W_{i} \subset W$ and whose positive dimensional cells correspond to the strata of $W$, that is, to irreducible components of intersections $\cap_{i \in J} W_{i}$ for some $J \subset I$; see Definition 7 .

In the papers [Kol11a, KK11, Kol12] many interesting singularities were obtained by constructing a simple normal crossing variety $W$ whose dual complex is topologically complicated and then realizing $W$ as the exceptional divisor of a (partial) resolution of a singularity.

The aim of this note is to prove the following existence result for simplicial complexes.

TheOREM 1. Let $\mathcal{C}$ be a finite simplicial complex of dimension $\leqslant n$. Then there exist a smooth projective variety $Y$ of dimension $n+1$ and a simple normal crossing divisor $D \subset Y$ such that $\mathcal{D}(D) \cong \mathcal{C}$. Moreover, we can achieve that either one (but not both) of the following conditions is also satisfied:

(i) $Y$ and all the strata of $D$ are rational.

(ii) $K_{Y}+D$ is ample.

This is stronger than the results in [KK11] in three aspects. We realize $\mathcal{C}$ up to isomorphism (not just up to homotopy equivalence) and $\operatorname{dim} D=\operatorname{dim} \mathcal{C}$ is optimal since $\operatorname{dim} W \geqslant \operatorname{dim} \mathcal{D}(W)$ for every simple normal crossing variety $W$. Furthermore, by construction $D$ is naturally a hypersurface in a smooth projective variety, while in earlier versions this was achieved only after a detailed study of a resolution process in [Kol12, Sec.6]. The last property is very helpful in constructing singularities. It simplifies the method of [Koll1a] and also leads to isolated singularities.

Received 28 March 2013, accepted in final form 14 August 2013.

2010 Mathematics Subject Classification 14J17, 14F35, $14 \mathrm{M} 12$

This journal is (C) Foundation Compositio Mathematica 2014. This article is distributed with Open Access under the terms of the Creative Commons Attribution Non-Commercial License, which permits non-commercial reuse, distribution, and reproduction in any medium, provided that the original work is properly cited. For commercial re-use, please contact the Foundation Compositio Mathematica.

I thank C. Xu and the referee for many comments and corrections. Partial financial support was provided by the NSF under grant number DMS-07-58275 and by the Simons Foundation. 


\section{JÁNOS KOLLÁR}

Theorem 2. Let $\mathcal{C}$ be a finite simplicial complex of dimension $\leqslant n$. Then there exist an isolated singularity $(x \in X)$ of dimension $n+1$ and a $\log$ resolution $\pi: Y \rightarrow X$ with exceptional divisor $E=\operatorname{Supp} \pi^{-1}(x)$ such that $\mathcal{D}(E) \cong \mathcal{C}$.

Both of the alternatives in Theorem 1 are useful. The first case can be utilized to complete the characterization of dual complexes of resolutions of isolated rational singularities started in [KK11, Kol12].

Theorem 3. For a finite simplicial complex $\mathcal{C}$, the following are equivalent:

(i) $\mathcal{C}$ is connected, $\operatorname{dim} \mathcal{C} \leqslant n$ and $H_{i}(\mathcal{C}, \mathbb{Q})=0$ for $i>0$.

(ii) There exist an isolated rational singularity $(x \in X)$ of dimension $n+1$ and a log resolution $\pi: Y \rightarrow X$ with exceptional divisor $E=\operatorname{Supp} \pi^{-1}(x)$ such that $\mathcal{D}(E) \cong \mathcal{C}$.

Let $(x \in X)$ be a (not necessarily isolated) singularity and let $g: Y \rightarrow X$ be a resolution such that $E:=\operatorname{Supp} g^{-1}(x) \subset Y$ is a simple normal crossing divisor. The dual complex $\mathcal{D}(E)$ depends on the choice of $Y$ but its homotopy type does not; see Definition 10. This homotopy type is denoted by $\mathcal{D} \mathcal{R}(x \in X)$. If $K_{X}$ is Cartier (or at least $\mathbb{Q}$-Cartier), then [dFKX12] identifies a distinguished homeomorphism class $\mathcal{D} \mathcal{M R}(x \in X)$ within the homotopy class $\mathcal{D} \mathcal{R}(x \in X)$; see Definition 11. Using the alternative Theorem 1(ii), we see that the homeomorphism classes $\mathcal{D} \mathcal{M R}(x \in X)$ can be arbitrary.

TheOrem 4. For every finite, connected simplicial complex $\mathcal{C}$ of dimension $\leqslant n$ there is a normal, isolated singularity $(x \in X)$ of dimension $n+1$ such that $K_{X}$ is Cartier and $\mathcal{D} \mathcal{M R}(x \in X)$ is homeomorphic to $\mathcal{C}$.

Open PROBlems 5. There are four main open problems in connection with dual complexes of varieties and of singularities.

(5.1) It is interesting to understand the possible relation between the dual complex $\mathcal{D}(X)$ and the canonical class $K_{X}$. If $K_{X}$ is ample, then $\mathcal{D}(X)$ can be arbitrary by Theorem 1 (ii). If $-K_{X}$ is ample, then $\mathcal{D}(X)$ is a simplex by [Fuj12a, Fuj12b]. The really interesting case seems to be when $K_{X} \sim 0$ or, more generally, when $K_{X} \sim_{\mathbb{Q}} 0$. These lead to log canonical singularities and their structure is not yet understood. By [Kol11b] (see also [Kol13, Sec.4.4]), in this case either $\operatorname{dim} \mathcal{D}(X)=1$ or every codimension 1 cell is the face of exactly two maximal dimensional cells. In all examples that I know of, $\mathcal{D}(X)$ is an orbifold.

(5.2) In many cases, the most economical realization of a homeomorphism type is given not by a simplicial complex but by a $\Delta$-complex in the terminology of [Hat02, p.103]. Thus it would be quite useful to know that every $\Delta$-complex can be realized as the dual complex of a normal crossing variety. This would give the optimal answer along the lines proposed by [Sim11]. See [Kap12] for very interesting related results.

(5.3) Usually one cannot satisfy both of the alternatives in Theorem 1 simultaneously. Indeed, if $v_{n-1} \in \mathcal{D}(D)$ is an $(n-1)$-cell that is the face of one or two $n$-cells, then the corresponding stratum is a smooth curve $C \subset D$ with one or two smaller strata on it. Thus if $C$ is rational, then $\left(K_{D} \cdot C\right)=-1$ or $\left(K_{D} \cdot C\right)=0$. This is, however, the only obstruction that I know.

(5.4) A shortcoming of our construction is that it is not local. Let $X=\cup_{i \in I} X_{i}$ be an snc variety. The link of a vertex $v_{i} \in \mathcal{D}(X)$ is determined by those $X_{j}$ for which $X_{j} \cap X_{i} \neq \emptyset$. Conversely, given a complex $\mathcal{C}$, one could hope to that there is a procedure to construct an snc variety $X=\cup_{i \in I} X_{i}$ such that $\mathcal{D}(D) \cong \mathcal{C}$ and each $X_{i}$ is determined by the link of $v_{i} \in \mathcal{C}$. Such a 
procedure exists if $\operatorname{dim} \mathcal{C}=2$; see [Kol11a]. The study of parasitic intersections in [KK11] suggests that this may be too much to hope for, but leaves open the possibility that $X_{i}$ should depend only on the $(\operatorname{dim} \mathcal{C}-1)$-times iterated link of $v_{i}$. One clearly needs a more local construction if one hopes to control the canonical class $K_{X}$.

\section{Dual complexes}

Since our main interest is in the topological aspects of these questions, we work with varieties over $\mathbb{C}$. However, everything applies to varieties over arbitrary fields. See [Kol13] for the more delicate aspects that appear when the base field is not algebraically closed and has positive characteristic.

DeFinition 6. Let $W$ be a variety with irreducible components $\left\{W_{i}: i \in I\right\}$. We say that $W$ is a simple normal crossing variety (abbreviated as snc) if the $W_{i}$ are smooth and every point $p \in W$ has an open (Euclidean) neighborhood $p \in U_{p} \subset W$ and an embedding $U_{p} \hookrightarrow \mathbb{C}^{n+1}$ such that the image of $U_{p}$ is an open subset of the union of coordinate hyperplanes $\left(z_{1} \cdots z_{n+1}=0\right)$. A stratum of $W$ is any irreducible component of an intersection $\cap_{i \in J} W_{i}$ for some $J \subset I$.

The intersection of any two strata is also a union of lower-dimensional strata. Thus the minimal strata are disjoint from each other and their union is smooth.

DeFinition 7. The combinatorics of a simple normal crossing variety $W$ is encoded by a cell complex $\mathcal{D}(W)$ whose vertices are labeled by the irreducible components of $W$; for every stratum $Z \subset \cap_{i \in J} W_{i}$, we attach a $(|J|-1)$-dimensional cell. Note that for any $j \in J$, there is a unique irreducible component of $\cap_{i \in J \backslash\{j\}} W_{i}$ that contains $Z$; this specifies the attaching map. The complex $\mathcal{D}(W)$ is called the dual complex or dual graph of $W$. Thus $\mathcal{D}(W)$ is a simplicial complex iff $\cap_{i \in J} W_{i}$ is irreducible (or empty) for every $J$. In general, $\mathcal{D}(W)$ is a regular cell complex; cf. [Hat02, p.534]. The minimal strata of $W$ correspond to the maximal cells of $\mathcal{D}(W)$.

It is clear that $\operatorname{dim} \mathcal{D}(W) \leqslant \operatorname{dim} W$ and that $\mathcal{D}(W)$ is connected iff $W$ is. More generally, the cohomology groups $H^{i}(\mathcal{D}(W), \mathbb{Q})$ can be identified with the weight 0 part of the Hodge structure on $H^{i}(W, \mathbb{Q})$; see [ABW13]. One can think of $\mathcal{D}(W)$ as the combinatorial part of the topology of $W$.

Definition 8. Let $X$ be a variety over $\mathbb{C}$ and let $D \subset X$ be a divisor. We say that $(X, D)$ is a normal crossing pair if $X$ is smooth and every point $x \in X$ has an Euclidean open neighborhood $x \in U_{x} \subset X$ with local coordinates $z_{1}, \ldots, z_{n}$ such that $D \cap U_{x}=\left(z_{1} \cdots z_{r}=0\right)$ for some $r$. We say that $(X, D)$ is a simple normal crossing pair if, in addition, the irreducible components $D_{i} \subset D$ are smooth.

If $X$ is smooth, then $(X, D)$ is a simple normal crossing pair iff $D$ is a simple normal crossing variety. (In Lemma 16 the key point is to understand what happens when $D$ is a simple normal crossing variety but $X$ is not smooth.) One can think of a simple normal crossing variety $D=$ $\cup_{i \in I} D_{i}$ as being glued together from the simple normal crossing pairs $\left(D_{i},\left.\sum_{j \neq i} D_{j}\right|_{D_{i}}\right)$. The difference between the two variants is technical but important for several of our results.

Construction 9. (Blowing up strata) Let $W$ be a simple normal crossing variety and let $Z \subset W$ be a stratum. Then the blow-up $B_{Z} W$ is again a simple normal crossing variety. To see this, take a local chart where

$$
(Z \subset W)=\left(\left(x_{1}=\cdots=x_{r}=0\right) \subset\left(x_{1} \cdots x_{n}=0\right)\right) \subset \mathbb{C}^{n+m} .
$$




\section{JÁNOS KOLLÁR}

The blow-up $B_{Z} \mathbb{C}^{n+m}$ is defined by

$$
B_{Z} \mathbb{C}^{n+m}=\left(x_{i} s_{j}=x_{j} s_{i}: 1 \leqslant i, j \leqslant r\right) \subset \mathbb{C}_{\mathbf{x}}^{n+m} \times \mathbb{P}_{\mathbf{s}}^{r-1}
$$

and the blow-up $B_{Z} W$ is defined by

$$
B_{Z} W=\left(s_{1} \cdots s_{r} \cdot x_{r+1} \cdots x_{n}=0\right) \subset B_{Z} \mathbb{C}^{n+m} .
$$

In a typical local chart $U_{r}:=\left(s_{r} \neq 0\right)$, we can use new coordinates

$$
\left(x_{1}^{\prime}=s_{1} / s_{r}, \ldots, x_{r-1}^{\prime}=s_{r-1} / s_{r}, s_{r}, x_{r+1}, \ldots, x_{n+m}\right)
$$

to obtain

$$
B_{Z} W \cap U_{r} \cong\left(x_{1}^{\prime} \cdots x_{r-1}^{\prime} \cdot x_{r+1} \cdots x_{n}=0\right) \subset \mathbb{C}^{n} .
$$

Let $v_{Z} \subset \mathcal{D}(W)$ be the cell corresponding to $Z$. We see from the above computations that $\mathcal{D}\left(B_{Z} W\right)$ is obtained from $\mathcal{D}(W)$ by removing all the cells that have $v_{Z}$ as a face, that is, removing the star of $v_{Z}$. In particular, if $Z$ is a minimal stratum, then we remove the cell $v_{Z}$.

Although we do not need it, it is useful to know that there is another way to blow up a stratum. If $(X, D)$ is a simple normal crossing pair and $Z \subset D$ is a stratum, one can consider the blow-up $\pi: B_{Z} X \rightarrow X$ with exceptional divisor $E_{Z}$. Then $B_{Z} D \cong \pi_{*}^{-1} D$ and $\left(B_{Z} X, E_{Z}+B_{Z} D\right)$ is again a simple normal crossing pair. It is not hard to see that $\mathcal{D}\left(E_{Z}+B_{Z} D\right)$ is obtained as the stellar subdivison of $\mathcal{D}(D)$ with vertex $v_{Z}$.

Definition 10. Let $X$ be a variety and let $x \in X$ be a point. Choose a resolution of singularities $\pi: Y \rightarrow X$ such that $E_{x}:=\operatorname{Supp} \pi^{-1}(x) \subset Y$ is a simple normal crossing divisor. We denote by $\mathcal{D}\left(E_{x}\right)$ the corresponding dual complex.

The dual graph of a normal surface singularity has a long history but systematic investigations in higher dimensions were started only recently; see [Thu07, Ste08, Pay13, ABW13]. It is proved in these papers that the homotopy type (even the simple homotopy type, see [Coh73, Chap.V]) of $\mathcal{D}\left(E_{x}\right)$ is independent of the resolution $Y \rightarrow X$. We denote it by $\mathcal{D} \mathcal{R}(x \in X)$, where $\mathcal{D} \mathcal{R}$ stands for the Dual complex of a Resolution. It is clear that $\operatorname{dim} \mathcal{D} \mathcal{R}(x \in X) \leqslant \operatorname{dim} X-1$ and that $\mathcal{D} \mathcal{R}(x \in X)$ is connected if $X$ is normal.

DEFINITION 11. It was observed in [dFKX12] that the dual complex of a divisor $D \subset Y$ is defined whenever $(Y, D)$ is divisorial log terminal (abbreviated as $d l t)$; see [KM98, 2.37] for the definition of dlt. If $(x \in X)$ is an isolated singularity and $K_{X}$ is $\mathbb{Q}$-Cartier, then there is a proper birational morphism $\pi: Y \rightarrow X$ such that $\left(Y, E_{Y}\right)$ is dlt and $K_{Y}+E_{Y}$ is $\pi$-nef, where $E_{Y}=\operatorname{Supp} \pi^{-1}(x)$. Such a morphism is called a dlt modification.

It is proved in [dFKX12] that

(i) $\mathcal{D}\left(E_{Y}\right)$ is homotopy equivalent to $\mathcal{D} \mathcal{R}(x \in X)$;

(ii) up to homeomorphism, $\mathcal{D}\left(E_{Y}\right)$ does not depend on the choice of $Y$.

Thus, for such singularities, $\mathcal{D}\left(E_{Y}\right)$ defines a distinguished homeomorphism class, denoted by $\mathcal{D} \mathcal{M} \mathcal{R}(x \in X)$, within the homotopy class $\mathcal{D} \mathcal{R}(x \in X)$; here $\mathcal{D} \mathcal{M} \mathcal{R}$ stands for the Dual complex of a Minimal partial Resolution. The hard part of [dFKX12] is the proof of part (i), but in our examples we check this directly (cf. Lemma 16).

\section{Construction of simple normal crossing varieties}

In [KK11] we constructed snc varieties $W=\cup_{i \in I} W_{i}$ by first obtaining the irreducible components $W_{i}$ and then gluing them together to get $W$. This had two technical disadvantages. First, the 


\section{SimPle NORMAL CROSSING VARIETIES}

projectivity of the resulting $W$ was hard to control and second, there was no natural embedding of $W$ into any smooth variety.

The process turns out to be much easier if we break the symmetry on the algebraic side but preserve it topologically. Given $\mathcal{C}$, first we construct an snc variety $X_{0}$ such that $\mathcal{D}\left(X_{0}\right)$ has the same dimension and the same vertex set as $\mathcal{C}$ but the maximum possible number of positive dimensional cells. Then we remove the excess positive dimensional cells one dimension at a time. A disadvantage is that we lose information about finite group actions, while the construction of [KK11] preserved symmetries. The key step is the following, where we use $\left(\begin{array}{l}I \\ r\end{array}\right)$ to denote the set of $r$-element subsets of the set $I$.

Lemma 12. Let $\mathcal{C}$ be a finite simplicial complex of dimension $n$ with vertices $\left\{v_{i}: i \in I\right\}$. Fix $0 \leqslant r<n$. Let $X_{r}$ be an snc variety such that $\mathcal{D}\left(X_{r}\right)$ is a simplicial complex and let $\tau_{r}: \mathcal{C} \hookrightarrow \mathcal{D}\left(X_{r}\right)$ be an embedding that is a bijection on the vertices and on cells of dimension $\geqslant n-r+1$. Let $J_{n-r+1} \subset\left(\begin{array}{c}I \\ n-r+1\end{array}\right)$ be those $(n-r+1)$-element subsets that do not span an $(n-r)$-cell in $\mathcal{C}$ and let $Z_{r} \subset X_{r}$ be the union of the strata that correspond to elements of $\tau_{r}\left(J_{n-r+1}\right)$. Then

(i) $Z_{r}$ is smooth;

(ii) $X_{r+1}:=B_{Z_{r}} X_{r}$, the blow-up of $Z_{r} \subset X_{r}$, is an snc variety;

(iii) $\mathcal{D}\left(X_{r+1}\right) \subset \mathcal{D}\left(X_{r}\right)$;

(iv) the restriction of $\tau_{r}$ gives an embedding $\tau_{r+1}: \mathcal{C} \hookrightarrow \mathcal{D}\left(X_{r+1}\right)$ which is a bijection on the vertices and on cells of dimension $\geqslant n-r$.

Proof. If $s_{n-r+1} \subset I$ does not span an $(n-r)$-cell in $\mathcal{C}$, then no subset of $I$ containing $s_{n-r+1}$ spans a cell in $\mathcal{C}$. Thus either $s_{n-r+1}$ does not span a cell in $\mathcal{D}\left(X_{r}\right)$ or it spans a maximal cell in $\mathcal{D}\left(X_{r}\right)$. Since maximal cells correspond to minimal strata, $Z_{r}$ is a union of minimal strata. The minimal strata are disjoint from each other, hence $Z_{r}$ is smooth and of pure codimension $n-r$. Thus, by Construction $9, X_{r+1}=B_{Z_{r}} X_{r}$ is an snc variety and $\mathcal{D}\left(X_{r+1}\right)$ is obtained from $\mathcal{D}\left(X_{r}\right)$ by removing all $(n-r)$-cells that do not correspond to a cell in $\mathcal{C}$.

The following yields a proof of Theorem 1(i).

Proposition 13. Let $\mathcal{C}$ be a finite simplicial complex of dimension $n$. Then there is an snc variety $X$ such that $\mathcal{D}(X) \cong \mathcal{C}, \operatorname{dim} X=\operatorname{dim} \mathcal{C}, X$ is a hypersurface in a smooth, projective variety $Y, X$ is defined over $\mathbb{Q}$ and both the strata of $X$ and $Y$ are rational varieties.

Proof. Let $\left\{v_{i}: i \in I\right\}$ be the vertices of $\mathcal{C}$. In $\mathbb{P}^{n+1}$, let $\left\{H_{i}: i \in I\right\}$ be hyperplanes in general position. One can get concrete examples by fixing distinct numbers $a_{i}$ and setting

$$
H_{i}=\left(x_{0}+a_{i} x_{1}+a_{i}^{2} x_{2}+\cdots+a_{i}^{n+1} x_{n+1}=0\right) \subset \mathbb{P}^{n+1} .
$$

No $n+2$ of the $H_{i}$ have a point in common since the coefficients form a Vandermonde matrix. If $a_{i} \in \mathbb{Q}$, then $H_{i}$ is defined over $\mathbb{Q}$. If the $a_{i}$ are conjugate over $\mathbb{Q}$, their union is defined over $\mathbb{Q}$ but has a nontrivial Galois action on its geometric irreducible components.

Set $X_{0}:=\cup_{i \in I} H_{i}$ and $Y_{0}:=\mathbb{P}^{n+1}$. Note that $X_{0}$ is an snc variety, all of its strata are linear spaces and for $0 \leqslant r \leqslant n$, the $(n-r)$-dimensional strata of $X_{0}$ are in a natural bijection with $\left(\begin{array}{c}I \\ r+1\end{array}\right)$.

Every subset $J \subset I$ with at most $n+1$ elements spans a cell in $\mathcal{D}\left(X_{0}\right)$, hence there is a natural embedding $\tau_{0}: \mathcal{C} \hookrightarrow \mathcal{D}\left(X_{0}\right)$ that is a bijection on vertices. Thus $X_{0}$ and $\tau_{0}$ satisfy the assumptions of Lemma 12 with $r=0$ since neither $\mathcal{C}$ nor $\mathcal{D}\left(X_{0}\right)$ contains any cells of dimension 


\section{JÁNOS KOLLÁR}

greater than $n$. Using Lemma 12 inductively for $0 \leqslant r<n$, we obtain embedded snc varieties $X_{r} \subset Y_{r}$ and smooth subvarieties $Z_{r} \subset X_{r}$ such that

$$
X_{r+1}=B_{Z_{r}} X_{r} \quad \text { and } \quad Y_{r+1}=B_{Z_{r}} Y_{r} .
$$

Furthermore, $\tau_{0}$ restricts to embeddings $\tau_{r}: \mathcal{C} \hookrightarrow \mathcal{D}\left(X_{r}\right)$ which are bijections on the vertices and on cells of dimension $\geqslant n-r+1$.

Finally, we get $X_{n}$ such that $\mathcal{C} \hookrightarrow \mathcal{D}\left(X_{n}\right)$ is a bijection on cells in every dimension. The strata of $X_{r}$ are obtained from linear subspaces in $Y_{0}:=\mathbb{P}^{n+1}$ by blowing up lower-dimensional linear subspaces, hence the strata are rational. Therefore $X:=X_{n}$ satisfies all the requirements.

Using the pair $X \subset Y$ obtained in Proposition 13 and the cyclic cover construction as in [KM98, 2.50], the following shows Theorem 1(ii).

Lemma 14. Let $Y$ be a smooth, projective variety of dimension $n+1$, let $D \subset Y$ be an snc divisor and let $\left\{p_{j}: j \in J\right\}$ be the 0-dimensional strata. Let $\tau: Y_{1} \rightarrow Y$ be a double cover ramified along a general, sufficiently ample divisor $H^{\prime} \sim 2 H$. For each $p_{j}$, pick a preimage $q_{j} \in \tau^{-1}\left(p_{j}\right)$ and let $\pi: Y_{2} \rightarrow Y_{1}$ be the blow-up of the points $\left\{q_{j}: j \in J\right\}$. Set $D_{1}:=\tau^{*} D$ and $D_{2}:=\pi_{*}^{-1} D_{1}$. Then

(i) $Y_{2}$ is a smooth, projective variety and $D_{2} \subset Y_{2}$ is an snc divisor;

(ii) $K_{Y_{2}}+D_{2}$ is ample;

(iii) the composite $\tau \circ \pi$ induces an isomorphism $\mathcal{D}\left(D_{2}\right) \cong \mathcal{D}(D)$.

Proof. By the Hurwitz formula,

$$
K_{Y_{1}}+D_{1} \sim_{\mathbb{Q}} \tau^{*}\left(K_{Y}+D+H\right),
$$

thus if $H$ is sufficiently ample, then $K_{Y_{1}}+D_{1}$ is the pull-back of a very ample line bundle from $Y$. (As for hyperelliptic curves, $K_{Y_{1}}+D_{1}$ is usually not very ample.) We may even arrange that

$$
\mathcal{O}_{Y}\left(K_{Y}+D+H\right)\left(-\sum_{j} p_{j}\right)
$$

is very ample on $Y \backslash\left\{p_{j}: j \in J\right\}$.

Let $E \subset Y_{2}$ be the exceptional divisor of $\pi$; it is a union of $|J|$ disjoint copies of $\mathbb{P}^{n}$. Then we have

and therefore

$$
K_{Y_{2}} \sim \pi^{*} K_{Y_{1}}+n E \text { and } D_{2} \sim \pi^{*} D_{1}-(n+1) E
$$

$$
K_{Y_{2}}+D_{2} \sim \pi^{*}\left(K_{Y_{1}}+D_{1}\right)-E .
$$

This shows that $K_{Y_{2}}+D_{2}$ is ample on $E$, and the fact that (14.1) is very ample on $Y \backslash\left\{p_{j}: j \in J\right\}$ implies that $K_{Y_{2}}+D_{2}$ is ample on $Y_{2} \backslash E \cong Y_{1} \backslash\left\{q_{j}: j \in J\right\}$. Thus $K_{Y_{2}}+D_{2}$ is ample.

Finally, let $W \subset D$ be a positive-dimensional stratum. By general position arguments, $\tau^{-1}(W)$ is smooth. Moreover, it is a double cover of $W$ ramified along the nonempty set $W \cap H^{\prime}$. Hence $\tau^{-1}(W)$ is irreducible. Thus $\tau_{*}$ is a bijection on the cells of dimension $<n$ but every $n$-dimensional cell of $\mathcal{D}(D)$ has two preimages in $\mathcal{D}\left(D_{1}\right)$. The blow-up of the points $q_{j}$ removes one of these cells, giving the isomorphism $\mathcal{D}\left(D_{2}\right) \cong \mathcal{D}(D)$.

\section{Construction of singularities}

Starting with a simplicial complex $\mathcal{C}$, in Theorem 1 we obtained an embedded snc variety $D \subset Y$ such that $\mathcal{D}(D) \cong \mathcal{C}$. If $D \subset Y$ can be contracted to a point, then we get an isolated singularity $(x \in X)$ one of whose log resolutions is $(D \subset Y)$. 


\section{SIMPLE NORMAL CROSSING VARIETIES}

In general, $D$ is not contractible; we have to perform some blow-ups first.

Lemma 15. Let $Y$ be a smooth variety, let $D \subset Y$ be an snc divisor and let $H \subset Y$ be another divisor intersecting $D$ transversally. Set $Z:=D \cap H$ and let $\pi: Y_{1}:=B_{Z} Y \rightarrow Y$ be the blow-up with exceptional divisor $E$. Let $D_{1}:=\pi_{*}^{-1} D$ denote the birational transform of $D$. Then

(i) $D_{1} \sim \pi^{*} D-E$ is a Cartier divisor;

(ii) $D_{1} \cong D$;

(iii) $Y_{1} \backslash D_{1}$ is smooth;

(iv) the singularities of $\left(Y_{1}, D_{1}\right)$ are analytically equivalent to

$$
\left(x_{1} \cdots x_{n}=z=0\right) \subset\left(x_{1} \cdots x_{n}=y z\right) \subset \mathbb{A}^{n+m+2} ;
$$

(v) $\left(Y_{1}, D_{1}\right)$ is dlt;

(vi) $\left.\left.N_{D_{1}, Y_{1}} \cong \mathcal{O}_{Y_{1}}\left(D_{1}\right)\right|_{D_{1}} \cong \mathcal{O}_{Y}(D-H)\right|_{D}$.

Proof. The first five assertions are local on $Y$, hence we may assume that there are local coordinates $y_{1}, \ldots, y_{n}$ such that $D=\left(y_{1} \cdots y_{r}=0\right)$ and $H=\left(y_{n}=0\right)$ for some $r<n$. The blow-up is given by an equation

$$
\left(y_{1} \cdots y_{r} \cdot s=y_{n} \cdot t\right) \subset Y \times \mathbb{P}_{s t}^{1} \quad \text { and } \quad D_{1}=\left(y_{1} \cdots y_{r}=t=0\right)=(t=0) .
$$

In the affine chart $t \neq 0$, we get the equation $y_{1} \cdots y_{r} \cdot(s / t)=y_{n}$, which shows that $Y_{1} \backslash D_{1}$ is smooth. The singularities of $\left(Y_{1}, D_{1}\right)$ are locally of the form

$$
\left(y_{1} \cdots y_{r}=v=0\right) \subset\left(y_{1} \cdots y_{r}=y_{n} \cdot v\right) \subset Y \times \mathbb{A}_{v}^{1} .
$$

This shows statement (iv). Statement (v) is proved in Lemma 16.

The normal bundle of $D_{1}$ is $\left.\mathcal{O}_{Y_{1}}\left(D_{1}\right)\right|_{D_{1}}$. Note that $D_{1} \sim \pi^{*} D-E$ and that $\pi_{*}^{-1} H \sim \pi^{*} H-E$ is disjoint from $D_{1}$. Thus

$$
\mathcal{O}_{Y_{1}}\left(D_{1}\right) \cong \mathcal{O}_{Y_{1}}\left(\pi^{*} D-\pi^{*} H\right) \cong \pi^{*} \mathcal{O}_{Y}(D-H) .
$$

Restricting to $D_{1} \cong D$ gives statement (vi).

Lemma 16. Let $Y$ be a normal variety and let $D \subset Y$ be a codimension 1 subvariety. Assume that $D$ is an snc variety and that at every singular point $x \in \operatorname{Sing} Y$, there are local analytic coordinates such that the pair $(Y, D)$ is given as a product of an snc pair with one of the form

$$
\left(\left(y_{1} \cdots y_{r}=y_{n+1}=0\right) \subset\left(y_{1} \cdots y_{r}=y_{n} y_{n+1}\right)\right) \subset \mathbb{A}^{n+1}
$$

for some $0 \leqslant r<n \leqslant \operatorname{dim} Y$. Then $(Y, D)$ has a small $\log$ resolution $\pi:\left(Y^{*}, D^{*}\right) \rightarrow(Y, D)$ and $\mathcal{D}\left(D^{*}\right)=\mathcal{D}(D)$. In particular, $(Y, D)$ is dlt.

Proof. Let $D_{0} \subset D$ be an irreducible component. Let $\pi_{0}: Y^{\prime} \rightarrow Y$ denote the blow-up of $D_{0}$. Then $\pi_{0}$ is an isomorphism at the points where $D_{0}$ is Cartier. At other points, in suitable local coordinates we have

$$
\left(D_{0} \subset D \subset Y\right) \cong\left(\left(y_{r}=y_{n+1}=0\right) \subset\left(y_{1} \cdots y_{r}=y_{n+1}=0\right) \subset\left(y_{1} \cdots y_{r}=y_{n} y_{n+1}\right)\right) .
$$

The blow-up is covered by two coordinate charts. In one of them we have the new coordinate $y_{r}^{\prime}=y_{r} / y_{n+1}$ and the local equations are

$$
\left(D^{\prime} \subset Y^{\prime}\right) \cong\left(\left(y_{n+1}=0\right) \subset\left(y_{1} \cdots y_{r-1} \cdot y_{r}^{\prime}=y_{n}\right)\right) .
$$




\section{JÁNOS KOLLÁR}

Thus, in this chart, $D^{\prime}$ has only one irreducible component and $\left(Y^{\prime}, D^{\prime}\right)$ is an snc pair. In the other chart, the new coordinate is $y_{n+1}^{\prime}=y_{n+1} / y_{r}$ and the local equations are

$$
\left(\left(y_{r}=0\right) \subset\left(y_{r}=0\right) \cup\left(y_{1} \cdots y_{r-1}=y_{n+1}^{\prime}=0\right) \subset\left(y_{1} \cdots y_{r-1}=y_{n} y_{n+1}^{\prime}\right)\right) .
$$

Thus the $y_{r}$-coordinate splits off as a direct factor and the remaining equation is

$$
\left(\left(y_{1} \cdots y_{r-1}=y_{n+1}^{\prime}=0\right) \subset\left(y_{1} \cdots y_{r-1}=y_{n} y_{n+1}^{\prime}\right)\right) .
$$

We also see that the dual complex of $D$ is unchanged.

Performing these blow-ups for every irreducible component of $D$, we end up with $\pi: Y^{*} \rightarrow Y$ such that $\left(Y^{*}, D^{*}:=\pi_{*}^{-1} D\right)$ is an snc pair and $\mathcal{D}\left(D^{*}\right)=\mathcal{D}(D)$. Since $\pi$ is small, the discrepancies are unchanged as we go from $(Y, D)$ to $\left(Y^{*}, D^{*}\right)$ by [KM98, 2.30]. Since $\pi$ does not contract any stratum of $D^{*}$, we see that $(Y, D)$ is dlt.

Remark 17. Note that the above resolution process requires an ordering of the irreducible components of $D$. Thus it does not apply if the simple normal crossing variety $D$ is replaced by a normal crossing variety. This makes the study of the dual complex of resolutions with normal crossing exceptional divisors quite a bit harder.

Proof of Theorem 2. Start with a simplicial complex $\mathcal{C}$ and use Theorem 1 to obtain an snc pair $D \subset Y$ such that $\mathcal{D}(D) \cong \mathcal{C}$. Let $H \subset Y$ be a smooth divisor intersecting $D$ transversally such that $H-D$ is ample. As in Lemma 15, let $\pi: Y_{1} \rightarrow Y$ be the blow-up of $Z:=D \cap H$ and set $D_{1}:=\pi_{*}^{-1} D$. By Lemma 15 (ii), $D_{1} \cong D$ and by Lemma 15 (vi), its normal bundle is $\left.N_{D_{1}, Y_{1}} \cong \mathcal{O}_{Y}(D-H)\right|_{D}$. Thus $N_{D_{1}, Y_{1}}^{-1}$ is ample. Therefore, by [Gra62, Satz.3.8] and [Art70, Cor.6.12.a], $D_{1} \subset Y_{1}$ can be contracted to a point, at least analytically or étale locally. That is, there is an analytic space (resp. an algebraic variety) $U_{1}$ containing $D_{1}$ with an open embedding (resp. an étale morphism) $g:\left(D_{1} \subset U_{1}\right) \rightarrow\left(D_{1} \subset Y_{1}\right)$ and a contraction morphism

$$
\begin{array}{ccc}
D_{1} & \subset & U_{1} \\
\downarrow & & \downarrow \pi \\
x & \in & X
\end{array}
$$

such that $X$ is normal, $U_{1} \backslash D_{1} \cong X \backslash\{x\}$ and $D_{1}=\operatorname{Supp} \pi^{-1}(x)$.

By Lemma $15(\mathrm{iv}), U_{1}$ is not smooth, but Lemma 16 shows that there is a log resolution

$$
\tau:\left(D_{2} \subset U_{2}\right) \rightarrow\left(D_{1} \subset U_{1}\right)
$$

such that $\mathcal{D}\left(D_{2}\right)=\mathcal{D}\left(D_{1}\right)$. Thus $\pi \circ \tau$ gives a resolution

$$
\begin{array}{lll}
D_{2} & \subset & U_{2} \\
\downarrow & & \downarrow \pi \circ \tau \\
x & \in & X
\end{array}
$$

This proves Theorem 2 .

Remark 18. Although, as we noted, the above construction does not seem to be compatible with symmetries of $\mathcal{C}$, it is usually possible to realize symmetries of $\mathcal{C}$ as Galois group actions on $D_{1}$ (though not on $D_{2}$ by Remark 17). This may help in understanding the algebraic fundamental group of links.

Proof of Theorem 3. The proof is essentially in [KK11, Lem.39]; see also [Kol12, Sec.8].

The implication (ii) $\Rightarrow$ (i) of Theorem 3 was established in the cited papers but the key ingredients are already contained in [GS75, pp.68-72] and [Ste83, 2.14]. 


\section{SimPle NORMAL CROSSING VARIETIES}

The converse statements are not fully proved in [Kol12, Sec.8], but the reason is that instead of our Theorem 1, weaker existence results were used. Using the proof of Theorem 2, the proof is even simpler. Let $\mathcal{C}$ be a $\mathbb{Q}$-acyclic simplicial complex. First, [Kol13, 3.63] shows that with $D$ as in Theorem 1(i) we have $H^{i}\left(D, \mathcal{O}_{D}\right)=0$ for $i>0$. Then [Kol13, 3.54] implies that for $H$ sufficiently ample, the singularity $(x \in X)$ obtained in (17.1) and (17.2) is rational.

Proof of Theorem 4. Start with a simplicial complex $\mathcal{C}$ and use Theorem 1 to obtain an embedded snc variety $D \subset Y$ such that $\mathcal{D}(D) \cong \mathcal{C}$.

As before, we blow up a subvariety of the form $D \cap H$ and then contract the birational transform of $D$ to obtain the required singularity $(x \in X)$. However, we have to choose $H$ with some care. By Theorem 1(ii), we may assume that $K_{Y}+D$ is ample. Choose $m \gg 1$ such that $m\left(K_{Y}+D\right)+D$ is very ample and let $H \sim m\left(K_{Y}+D\right)+D$ be a smooth divisor that intersects $D$ transversally. Let $\pi: Y_{1} \rightarrow Y$ be the blow-up of $Z:=D \cap H$ with exceptional divisor $E$. Then we have

$$
K_{Y_{1}} \sim \pi^{*} K_{Y}+E, \quad D_{1}:=\pi_{*}^{-1} D \sim \pi^{*} D-E \quad \text { and } \quad \pi_{*}^{-1} H \sim \pi^{*} H-E,
$$

and therefore

$$
m K_{Y_{1}}+(m+1) D_{1} \sim \pi^{*}\left(m K_{Y}+(m+1) D\right)-E \sim \pi_{*}^{-1} H .
$$

Let $\left(D_{1} \subset U_{1}\right) \rightarrow\left(D_{1} \subset Y_{1}\right)$ be any analytic or étale neighborhood of $D_{1}$ whose image is disjoint from $\pi_{*}^{-1} H$. Then (18.1) implies that

$$
\mathcal{O}_{U_{1}}\left(m K_{U_{1}}+(m+1) D_{1}\right) \cong \mathcal{O}_{U_{1}} .
$$

Let $g:\left(D_{1} \subset U_{1}\right) \rightarrow\left(x_{1} \in X_{1}\right)$ be the contraction of $D_{1}$ to a normal singularity. Then the isomorphism (18.2) pushes forward to an isomorphism

$$
\mathcal{O}_{X_{1}}\left(m K_{X_{1}}\right) \cong g_{*} \mathcal{O}_{U_{1}}\left(m K_{U_{1}}+(m+1) D_{1}\right) \cong g_{*} \mathcal{O}_{U_{1}} \cong \mathcal{O}_{X_{1}} .
$$

Thus $m K_{X_{1}}$ is Cartier. Furthermore, $\left(U_{1}, D_{1}\right)$ is dlt by Lemma $15(\mathrm{iv})$ and

$$
\left.\left.\mathcal{O}_{U_{1}}\left(K_{U_{1}}+D_{1}\right)\right|_{D_{1}} \cong \mathcal{O}_{D_{1}}\left(K_{D_{1}}\right) \cong \mathcal{O}_{D}\left(K_{D}\right) \cong \mathcal{O}_{Y}\left(K_{Y}+D\right)\right|_{D}
$$

is ample by assumption, hence $g: U_{1} \rightarrow X_{1}$ is a dlt modification. Therefore, as noted in Definition $11, \mathcal{D} \mathcal{M} \mathcal{R}\left(x_{1} \in X_{1}\right)$ is homeomorphic to $\mathcal{D}\left(D_{1}\right) \cong \mathcal{D}(D) \cong \mathcal{C}$. This gives a requisite example where the canonical class is $\mathbb{Q}$-Cartier. From this we get an example where the canonical class is Cartier as follows. The isomorphism $\mathcal{O}_{X_{1}}\left(m K_{X_{1}}\right) \cong \mathcal{O}_{X_{1}}$ determines a degree $m$ cyclic cover

$$
\tau:(x \in X) \rightarrow\left(x_{1} \in X_{1}\right)
$$

(see [KM98, 2.49-53] for the construction and basic properties of cyclic covers.) Let $\pi:\left(U_{2}, D_{2}\right) \rightarrow$ $\left(U_{1}, D_{1}\right)$ be the $\log$ resolution constructed in Lemma 16 such that $\mathcal{D}\left(D_{2}\right) \cong \mathcal{D}\left(D_{1}\right)$. Corresponding to $X \rightarrow X_{1}$, we have a cyclic cover $\tau_{U}: U \rightarrow U_{2}$ that comes from the isomorphism

$$
\mathcal{O}_{U_{2}}\left(K_{U_{2}}+D_{2}\right)^{\otimes m} \sim \mathcal{O}_{U_{2}}\left(-D_{2}\right) \text {. }
$$

This shows that $D:=\operatorname{Supp} \tau_{U}^{-1}\left(D_{2}\right) \rightarrow D_{2}$ is an isomorphism. In particular, $U$ and $X$ are irreducible. The divisor $K_{U}+D$ is not relatively ample anymore, but $\left.\left(K_{U}+D\right)\right|_{D} \sim K_{D} \sim K_{D_{2}}$ is the pull-back of $\left.K_{D_{1}} \sim\left(K_{U_{1}}+D_{1}\right)\right|_{D_{1}}$, hence $K_{U}+D$ is nef over $X$.

The singularities of $D \subset U$ are locally of the form

$$
\left(\left(y_{1} \cdots y_{r}=y_{n}=0\right) \subset\left(y_{1} \cdots y_{r}=y_{n}^{m}\right)\right) \subset \mathbb{A}^{n+1}
$$




\section{JÁNOS KOLLÁR}

for some $2 \leqslant r<n$. These pairs are not dlt, but they are quotients of dlt pairs. Indeed, they can be written as

$$
\left(\left(u_{1} \cdots u_{r}=0\right) \subset \mathbb{A}_{\mathbf{u}}^{n}\right) / G_{m}
$$

where $y_{1}=u_{1}^{m}, \ldots, y_{r}=u_{r}^{m}, y_{n}=u_{1} \cdots u_{r}$ and $G_{m}$ is the group of diagonal matrices

$$
\left\{\operatorname{diag}\left(a_{1}, \ldots, a_{r}, 1, \ldots, 1\right): a_{1}^{m}=\cdots=a_{r}^{m}=a_{1} \cdots a_{r}=1\right\} .
$$

Thus, using $\left[\mathrm{dFKX} 12\right.$, Cor.38], $\mathcal{C} \cong \mathcal{D}\left(D_{1}\right) \cong \mathcal{D}\left(D_{2}\right) \cong \mathcal{D}(D)$ is homeomorphic to $\mathcal{D} \mathcal{M} \mathcal{R}(x \in X)$.

\section{Fundamental groups of rational links}

A group $G$ is called $\mathbb{Q}$-superperfect if $H_{1}(G, \mathbb{Q})=H_{2}(G, \mathbb{Q})=0$, see [KK11, Def.40]. By [KK11, Thm.42], for every finitely presented, $\mathbb{Q}$-superperfect group $G$ there is a 6 -dimensional rational singularity $(x \in X)$ such that $\pi_{1}(\operatorname{Lk}(x \in X))$, the fundamental group of the link of $(x \in X)$, is isomorphic to $G$. Our results also yield such examples in dimensions 4 and 5 . In addition, we get isolated singularities.

Theorem 19. Let $G$ be a finitely presented, $\mathbb{Q}$-superperfect group. For $n \geqslant 4$, there is an $n$-dimensional, isolated, rational singularity $(x \in X)$ such that

$$
\pi_{1}(\operatorname{Lk}(x \in X)) \cong \pi_{1}(\mathcal{D} \mathcal{R}(x \in X)) \cong G .
$$

Proof. By Lemma 21, there is a 3 -dimensional, $\mathbb{Q}$-acyclic, simplicial complex $\mathcal{C}$ such that $\pi_{1}(\mathcal{C}) \cong$ $G$. Thus, for $n \geqslant 4$, Theorem 3 constructs an $n$-dimensional rational singularity $(x \in X)$ and a log resolution $\pi: Y \rightarrow X$ with exceptional divisor $E$ such that $\pi_{1}(\mathcal{D} \mathcal{R}(x \in X))=\pi_{1}(\mathcal{D}(E)) \cong G$.

If we choose $H$ to be ample in the proof of Theorem 2, then, by [KK11, Thm.35], for the resulting singularity $(x \in X)$ there is a surjection

$$
\pi_{1}(\operatorname{Lk}(x \in X)) \rightarrow \pi_{1}(\mathcal{D} \mathcal{R}(x \in X))
$$

whose kernel is finite, cyclic and generated by any loop around an irreducible component of $E$. I do not have a formula for the order of the kernel, but the following argument shows how to modify the construction to ensure that (19.1) is an isomorphism.

Assume that there is a smooth rational curve $C \subset E$ such that $E$ is smooth along $C$. The boundary $L$ of the normal disc bundle of $E \subset Y$ restricted to $C$ is a circle bundle over $\mathbb{C P}^{1}$ with Chern class $(C \cdot E)$. Thus $\pi_{1}(L)$ is generated by a loop around the irreducible component of $E$ containing $C$ and $\left|\pi_{1}(L)\right|=-(C \cdot E)$. Thus, if we find two such rational curves $C_{i} \subset E$ such that $\left(C_{1} \cdot E\right)$ and $\left(C_{2} \cdot E\right)$ are relatively prime, then (19.1) is an isomorphism.

Our construction usually gives a large supply of such rational curves. For instance, assume that $\mathcal{C}$ contains three vertices $v_{1}, v_{2}, v_{3}$ such that no two are on the same 1 -simplex. (This can always be arranged by taking a barycentric subdivision.) Correspondingly, in Proposition 13, we have hyperplanes $H_{1}, H_{2}, H_{3} \subset \mathbb{P}^{n+1}$ and at some point we blow up the (birational transform of the) triple intersection $H_{1} \cap H_{2} \cap H_{3}$ and then the (birational transforms of the) pairwise intersections $H_{i} \cap H_{j}$. Following the method in Lemma 15, we have $\sum D_{i} \subset Y$, where $D_{i}$ is obtained from $H_{i}$ by repeated blow-ups. As we noted above, the exceptional divisor of the last blow-up is a $\mathbb{P}^{1}$-bundle; let $C_{i} \subset D_{i}$ be a typical fiber. Note that, by construction, $\left(C_{i} \cdot D_{i}\right)=-1$ and $\left(C_{i} \cdot D_{j}\right)=0$ for $i \neq j$.

Finally, we need to make a good choice of $H$ in Lemma 15. First choose $H_{0}$ such that $a=\left(C_{i} \cdot H_{0}\right)$ is independent of $i=1,2,3$. For $m \gg 1, H:=m H_{0}+D_{1}$ is still very ample, but 


\section{SIMPLE NORMAL CROSSING VARIETIES}

now $\left(C_{1} \cdot H\right)=m a-1$ and $\left(C_{2} \cdot H\right)=m a$. Thus, after the blow-up in Lemma 15 , we get two rational curves $C_{i} \subset E$ such that $\left(C_{1} \cdot E\right)$ and $\left(C_{2} \cdot E\right)$ are relatively prime.

Remark 20. As discussed in [KK11, Sec.7], if $(x \in X)$ is a 3-dimensional rational singularity, then $\pi_{1}(\mathcal{D} \mathcal{R}(x \in X))$ has a balanced presentation; that is, the number of relations equals the number of generators. For arbitrary rational singularities, the map (19.1) can have a very large kernel which is very hard to control in general. It is not known which groups can occur as fundamental groups of links of rational singularities in dimension 3 or higher.

The following result is well known.

Lemma 21. Let $G$ be a finitely presented group.

(i) There is a 2-dimensional simplicial complex $\mathcal{C}_{2}$ such that $\pi_{1}\left(\mathcal{C}_{2}\right) \cong G$.

(ii) There is a 3-dimensional simplicial complex $\mathcal{C}_{3}$ such that

$$
\pi_{1}\left(\mathcal{C}_{3}\right) \cong G, \quad H_{i}\left(\mathcal{C}_{3}, \mathbb{Z}\right) \cong H_{i}(G, \mathbb{Z}) \text { for } i=1,2 \text { and } H_{3}\left(\mathcal{C}_{3}, \mathbb{Z}\right) \cong 0 .
$$

(iii) If $G$ is $\mathbb{Q}$-superperfect, then $\mathcal{C}_{3}$ is $\mathbb{Q}$-acyclic.

Proof. Let $\left\langle a_{1}, \ldots, a_{r} \mid \omega_{1}, \ldots, \omega_{s}\right\rangle$ be a presentation of $G$. Start with $\mathcal{C}_{1}$, a bouquet of $r$ circles. If $\omega_{i}$ has word length $l_{i}$, we can kill it in $\pi_{1}\left(\mathcal{C}_{1}\right)$ by attaching an $l_{i}$-gon to $\mathcal{C}_{1}$ appropriately. We can think of the $l_{i}$-gon as made up of $l_{i}$ triangles. This way we have a 2 -dimensional $\Delta$-complex $\mathcal{C}_{2}$ whose fundamental group is $G$. Note that $H_{2}\left(\mathcal{C}_{2}, \mathbb{Z}\right)$ is free.

Let $\beta_{1}, \ldots, \beta_{t} \in H_{2}\left(\mathcal{C}_{2}, \mathbb{Z}\right)$ be a basis of the image of the Hurewicz map $\pi_{2}\left(\mathcal{C}_{2}\right) \rightarrow H_{2}\left(\mathcal{C}_{2}, \mathbb{Z}\right)$. We attach 3-balls $B_{i}$ to the $\beta_{i}$ to obtain a 3 -dimensional $\Delta$-complex $\mathcal{C}_{3}$. By construction, $\pi_{1}\left(\mathcal{C}_{3}\right) \cong$ $G$ (hence also $\left.H_{1}\left(\mathcal{C}_{3}, \mathbb{Z}\right) \cong H_{1}(G, \mathbb{Z})\right)$ and $H_{3}\left(\mathcal{C}_{3}, \mathbb{Z}\right)=0$. By Hopf's theorem [Hop42],

$$
H_{2}(G, \mathbb{Z}) \cong \operatorname{coker}\left[\pi_{2}\left(\mathcal{C}_{2}\right) \rightarrow H_{2}\left(\mathcal{C}_{2}, \mathbb{Z}\right)\right] \cong H_{2}\left(\mathcal{C}_{3}, \mathbb{Z}\right)
$$

which completes the proof of statement (ii). If $G$ is $\mathbb{Q}$-superperfect, then $H_{i}\left(\mathcal{C}_{3}, \mathbb{Q}\right)=0$ for $i=1,2$, hence $\mathcal{C}_{3}$ is $\mathbb{Q}$-acyclic.

The construction gives only a $\Delta$-complex, but its second barycentric subdivison is simplicial.

\section{REFERENCES}

ABW13 D. Arapura, P. Bakhtary and J. Włodarczyk, Weights on cohomology, invariants of singularities, and dual complexes, Math. Ann. 357 (2013), 513-550. http://dx.doi.org/10.1007/ s00208-013-0912-7

Art70 M. Artin, Algebraization of formal moduli. II. Existence of modifications, Ann. of Math. (2) 91 (1970), 88-135.

Coh73 M. M. Cohen, A course in simple-homotopy theory, Graduate Texts in Mathematics, Vol. 10, Springer-Verlag, New York (1973).

dFKX12 T. de Fernex, J. Kollár, and C. Xu, The dual complex of singularities, arXiv:1212.1675 (2012).

Fuj12a K. Fujita, Simple normal crossing Fano varieties and log Fano manifolds, arXiv:1206.1994 (2012).

Fuj12b - The Mukai conjecture for log Fano manifolds, arXiv:1206.2475 (2012).

Gra62 H. Grauert, Über Modifikationen und exzeptionelle analytische Mengen, Math. Ann. 146 (1962), $331-368$. 


\section{JÁNOS KOLLÁR}

GS75 Ph. Griffiths and W. Schmid, Recent developments in Hodge theory: a discussion of techniques and results, Discrete subgroups of Lie groups and applicatons to moduli (Internat. Colloq., Bombay, 1973), Oxford Univ. Press, Bombay (1975), 31-127.

Hat02 A. Hatcher, Algebraic topology, Cambridge University Press, Cambridge (2002).

Hop42 H. Hopf, Fundamentalgruppe und zweite Bettische Gruppe, Comment. Math. Helv. 14 (1942), 257-309.

Kap12 M. Kapovich, Dirichlet fundamental domains and complex-projective varieties, arXiv:1201.3129 (2012).

KK11 M. Kapovich and J. Kollár, Fundamental groups of links of isolated singularities, Journal AMS (to appear), arXiv:1109.4047 (2011).

KM98 J. Kollár and S. Mori, Birational geometry of algebraic varieties, Cambridge Tracts in Mathematics, 134, Cambridge University Press, Cambridge (1998); with the collaboration of C. H. Clemens and A. Corti; translated from the 1998 Japanese original.

Kol11a J. Kollár, New examples of terminal and log canonical singularities, arXiv:1107.2864 (2011).

Kol11b_ Sources of log canonical centers, arXiv:1107.2863 (2011).

Kol12 L Links of complex analytic singularities, arXiv:1209.1754 (2012).

Kol13 S Singularities of the minimal model program, Cambridge Tracts in Mathematics 200, Cambridge University Press, Cambridge (2013); with the collaboration of S. Kovács.

Pay13 S. Payne, Boundary complexes and weight filtrations, Michigan Math. J. 62 (2013), no. 2, 293322. http://dx.doi.org/10.1307/mm j/1370870374

Sim11 C. Simpson, Local systems on proper algebraic V-manifolds, Pure Appl. Math. Q. 7 (2011), 1675-1759. http://dx.doi.org/10.4310/PAMQ.2011.v7.n4.a27

Ste83 J. H. M. Steenbrink, Mixed Hodge structures associated with isolated singularities, Singularities, Part 2 (Arcata, Calif., 1981), Proc. Sympos. Pure Math., 40, Amer. Math. Soc., Providence, RI (1983), 513-536.

Ste08 D. A. Stepanov, A note on resolution of rational and hypersurface singularities, Proc. Amer. Math. Soc. 136 (2008), no. 8, 2647-2654. http://dx.doi.org/10.1090/ S0002-9939-08-09289-7

Thu07 A. Thuillier, Géométrie toroïdale et géométrie analytique non archimédienne. Application au type d'homotopie de certains schémas formels, Manuscripta Math. 123 (2007), no. 4, 381-451.

János Kollár kollar@math.princeton.edu

Princeton University, Princeton, NJ 08544-1000, USA 